\title{
The molecular mechanism and effect of cannabinoid-2 receptor agonist on the blood-spinal cord barrier permeability induced by ischemia-reperfusion injury
}

Ming-chao Yang, Hang-zhou Zhang, Zhe Wang, Fu-li You, Yan-feng Wang ${ }^{*}$

Department of Sports Medicine and Joint Surgery. The First Affiliated Hospital, China Medical University, Shen yang 110001, Liaoning Province, P.R. China

The final number of the text pages of the whole manuscript is 17 , and the number of figures is 10 .

*Corresponding Author: Yan-feng Wang

E-mail: wyfdoctor_2015@163.com 


\title{
The molecular mechanism and effect of cannabinoid-2 receptor agonist on the blood-spinal cord barrier permeability induced by ischemia-reperfusion injury
}

\begin{abstract}
Previous studies have shown that modulation of the receptor-mediated endocannabinoid system during ischemia injury can induce potent neuroprotective effects. However, little is known about whether cannabinoid-2 (CB2) receptor agonist would produce a protective effect on blood-spinal cord barrier (BSCB) during ischemia. Using an in vivo transient spinal cord ischemia model in rats, JWH-015 (1 $\mathrm{mg} / \mathrm{kg}$, i.p.), a CB2 receptor selective agonist, or vehicles were injected 20 minutes before ischemia. The effects of JWH-015 on BSCB permeability, the major structural protein for the formation of caveolae, caveolin-1 (cav-1), tight junction (TJ) protein Occludin and zona occludens protein-1 (ZO-1) were examined at day 1, day 3 and day 7 of reperfusion after transient spinal cord ischemia in rats. Here we demonstrated that JWH-015 significantly down-regulated the expression of cav-1, up-regulated the expression of TJ proteins, and then decreased the permeability of BSCB compared with control group. In addition, using an in vitro BBB model, oxygen glucose deprivation (OGD) was applied to simulate spinal cord ischemia in vitro in Human brain microvascular endothelial cells (HBMECs). JWH-015 greatly increased the transepithelial electrical resistance (TEER) and changed the distribution of ZO-1 and Occludin. Moreover, JWH-015 induced the expression of p-PKB and p-FoxO1 protein and decreased the expression of cav-1, which were greatly reversed by ROS inhibitor or PI3K inhibitor. Taken together, all of these results suggested that JWH-015 might regulate the BSCB permeability and this effect could be related to paracellular and transcellular pathway. And pharmacological CB2R ligands offer a new strategy for BSCB protection during ischemic injury.
\end{abstract}

Keywords: Blood-spinal cord barrier, Tight junction, Cannabinoid-2 receptor, Caveolin-1; Spinal cord ischemia reperfusion injury 


\section{Introduction}

Spinal cord ischemia reperfusion injury (SCII) is a kind of severe injury from central nervous system. SCII is not only acute neurodegenerative diseases, but also could alter blood-spinal cord barrier (BSCB) function eventually lead to impaired neuronal signaling and cognition (Li et al., 2015). Therefore, studies on the regulatory mechanism of BSCB after SCII will provide new ideas for the prevention of ischemic diseases.

Evidence from basic pharmacological studies has shown that endocannabinoid system has been under investigation as a potential target for neuroprotection and it could protect against ischemic stroke (England et al., 2014). Studies demonstrate that it plays an important modulatory role in normal blood brain barrier (BBB) physiology, and also affords a protection to the BBB during ischemic stroke (Hind et al., 2015). Recently, a possible connection between the cannabinoid receptors ( $\mathrm{CB}$ receptors) and the BBB also first gained attention. Hind et al. demonstrated that endocannabinoids modulate human BBB permeability in vitro (Hind et al., 2015). Pharmacological data have indicated that CB2 agonists are neuroprotective in cerebral ischemia (Choi et al., 2013) and increased transendothelial electrical resistance and the amount of tight junction protein present in membrane fractions during neuroinflammation (Ramirez et al., 2012), suggesting that CB2 receptor activation holds limitless promise as a practical and therapeutic intervention. However, it is unclear by which mechanism and the effect of $\mathrm{CB} 2$ receptor activation on $\mathrm{BSCB}$ during ischemia-reperfusion injury.

Caveolin-1, the major structural protein required for the formation of caveolae, is also highly necessary in regulating the activity and localization of signal molecules that are involved in vesicle fission (Mougeolle et al., 2015). The study confirmed that caveolin-1 are closely involved in the regulation of the permeability of BBB and directly regulate the expression of TJ protein and the number of pinocytosis vesicles (i.e. the paracellular pathway and transcellular pathway) (Zhao et al., 2014). In several models of adult brain injuries, the level of expression of caveolin-1 protein is increased in the endothelium in several animal models such as cold cortical injury 
(Nag et al., 2007), brain ischemia (Huang et al., 2012) and experimental spinal cord injury (Shin et al., 2005). And an increase of caveolin-1 in some brain injury models was related to BBB/BSCB breakdown (Nag et al., 2007). Altered expression of these TJ proteins could cause BBB breakdown and then result in edema formation after brain injury (Blixt et al., 2015). Both Occludin and ZO-1 are organized within the TJ by association with caveolin-1 in detergent-insoluble glycolipid rafts, membrane specializations closely related to caveolae. Caveolin-1 appears to constitute an early and critical modulator that leads to the disruption of TJ (Zhong et al., 2008). To investigate the mechanism and effect the $\mathrm{CB} 2$ receptor agonist pretreatment on $\mathrm{BSCB}$ after SCII, we utilized in vivo and in vitro ischemia model and evaluated the changes of BSCB permeability, the expression of TJ proteins, caveolin-1 protein, the level of FoxO1 phosphorylation and PKB phosphorylation in different time point group.

\section{Results}

\subsection{JWH-015 pretreatment improved the permeability of BSCB in SCII model} in rats

In the present study, the permeability of BSCB was evaluated by Evans blue leakage. The spinal cord tissue after SCII was stained in blue. Evans blue content and scope of blue staining in the spinal cord tissue was significantly decreased by JWH-015 pretreatment compared with SCII group (Fig. 1A). The Evans blue content of spinal cord tissue at day 1 , day 3 and day 7 after SCII is $4.8 \pm 0.4,4.2 \pm 0.5,3.5 \pm 0.2$, respectively. And they are 3.0 $\pm 0.3,2.8 \pm 0.2,2.5 \pm 0.2$ in JWH-015 group at day 1, day 3 and day 7 after SCII. There is a strong red fluorescence surrounding the vascular of in gray matter of spinal cord after SCII, which could be significantly reduced by JWH-015 pretreatment (Fig. 1B-F).

\subsection{JWH-015 pretreatment prevented the alteration of Occludin, ZO-1 and}

\section{Caveolin-1 induced by SCII in rats}

The expression of TJ proteins Occludin and ZO-1 were measured by western blots at different time point after SCII in rats. The results showed that the expression levels of TJ proteins Occludin and ZO-1 in spinal cord tissue were significantly decreased at 1 day, 3 day and 7 day after reperfusion (Fig. 2A). Moreover, JWH-015 partially 
prevented the down-regulation of Occludin and ZO-1 induced by SCII (Fig. 2A). The IDV ratio of Occludin for western blots at day 1, day 3 and day 7 after SCII is 0.2 $\pm 0.03,0.4 \pm 0.04,0.3 \pm 0.042$.(Fig. $2 \mathrm{~B}$ and C). And the IDV is $0.8 \pm 0.07,0.85 \pm 0.09$, $0.9 \pm 0.1$ at day 1 , day 3 and day 7 after SCII with JWH-015 pretreatment. And there was no significant difference in Sham-operated group and JWH-015+Sham-operated group at day 1, day 3 and day 7 (Fig. 2D-F). In addition, the expression and location of TJ proteins Occludin and ZO-1 were evaluated by immunohischemistry at different time point after SCII in rats. The effects of SCII-induced reduction of Occludin and ZO-1 in the vascular of the gray matter were attenuated by pretreatment with JWH-015 at day 1, day 3 and day 7 after reperfusion (Fig. 3 and Fig. 4).The mean optical density in SCII group and JWH-015+SCII group were shown in Fig.3G and Fig. 4G. In addition, Western blot analysis showed that the expression level of caveolin-1 protein in spinal cord tissue were significantly induced at day 1, day 3 and day 7 after SCII (Fig. 5). Moreover, JWH-015 pretreatment greatly prevented the up-regulation of caveolin-1 induced by SCII (Fig.5). The IDV ratios of caveolin-1 for western blot were shown in (Fig. $5 \mathrm{~B}$ and D).

\subsection{JWH-015 pretreatment improved the permeability of in vitro BBB}

In the study, the permeability of BBB in vitro was measured by TEER assays and HRP flux. OGD time-dependently reduced TEER across a monolayer of HBMECs. Furthermore, this effect of OGD was prevented by incubation of the cells with the JWH-015 for 20 min before exposure to OGD. The effects of JWH-015 on TEER were more significant in $10 \mu \mathrm{M}$ and $100 \mu \mathrm{M}$ groups than in the $1 \mu \mathrm{M}$ group (Fig 6A, $\left.{ }^{\#} P<0.05\right)$. JWH-015 at the dose of $10 \mu \mathrm{M}$ was administrated in the following experiments. We next determined the paracellular permeability toward HRP. Addition of JWH-015 to a HBMECs monolayer on Transwell filters reduced the leakage of HRP (Fig 6B, ${ }^{* *} P<0.01$ ) induced by OGD. These results have shown that JWH-015 pretreatment could greatly decrease the permeability of BBB in vitro.

\subsection{JWH-015 pretreatment prevented the OGD-Induced Alteration of ZO-1, Occludin and Caveolin-1 protein}

Treatment of HBMECs monolayers with OGD resulted in a significant reduction of 
Occludin and ZO-1 expression. However, the effects of OGD on the expression of Occludin and ZO-1 were attenuated by JWH-015 pretreatment (Fig.7). Immunofluorescence analysis revealed, after OGD administration, the immunoreactivities of Occludin and $\mathrm{ZO}-1$ were attenuated, and they were discontinuously distributed in the cellular boundaries, and immunofluorescence was observed partially in cytoplasmic (Fig.8A and C). JWH-015 pretreatment could significantly upregulate the expression and distribution of ZO-1 and Occludin in the cellular boundaries (Fig. $8 \mathrm{~B}$ and D). In addition, the expression of caveolin-1 was significantly increased by OGD, which were significantly attenuated by JWH-015 pretreatment (Fig. $9 \mathrm{~A}$ and B).

\subsection{JWH-015 pretreatment increased an activation of PKB phosphorylation induced by OGD}

To investigate the effect of JWH-015 on PKB activation in HBMECs, we evaluated the phosphorylation state of PKB by Western blotting. Phosphorylation of PKB is associated with activation of PKB signaling. Our results suggest that expression of phosphor-PKB in the JWH-015 pretreatment $(10 \mu \mathrm{M})$ group was greatly higher than in the OGD group. However, pretreatment of MPG or LY294002 partly prevented the phosphorylation of PKB induced by JWH015 (Fig. 10A). The IDV ratios of p-PKB/PKB for western blot were shown in Fig. $10 \mathrm{~B}$.

\subsection{JWH-015 increased an activation of FoxO1 phosphorylation induced by}

\section{OGD}

To investigate the effect of JWH-015 on FoxO1 activation in HBMECs, we evaluated the phosphorylation state of FoxO1 by Western blotting. Phosphorylation of FoxO1 is associated with activation of PKB signaling. Our result has shown that the protein expression of phosphor-FoxO1/FoxO1 in the JWH-015+OGD group is significantly enhanced compared to the OGD group (Fig. 10A). However, pretreatment of MPG or LY294002 partly prevented the phosphorylation of FoxO1 induced by JWH015. The IDV ratios of p-FoxO1/FoxO1 for western blot were shown in Fig. 10 B.

\section{Discussion}


Studies have demonstrated that modulation of the receptor-mediated endocannabinoid system during ischemia injury can produce potent neuroprotective effects (Arevalo-Martin et al., 2012). Recently Yu et al. demonstrated that post-stroke treatment with a CB2R agonist cannot efficiently suppress brain damages in stroke rats. However, pretreatment of $\mathrm{CB} 2 \mathrm{R}$ agonist significantly reduced the area of infarction in stroke animals (Yu et al., 2015), suggesting that early pretreatment with CB2 agonists reduced ischemic brain injury. The increase of BBB permeability induced by ischemic damage is one of key factors associated with neurodegeneration (Moretti et al., 2015). Here we demonstrated that JWH-015 could greatly down-regulate the expression of caveolin-1, up-regulate the expression of TJ proteins, and decrease the permeability of BSCB induced by SCII. In addition, using an in vitro BBB model, oxygen glucose deprivation (OGD) was applied to simulate spinal cord ischemia in vitro. JWH-015 could greatly increase the transepithelial electrical resistance (TEER) and change the distribution of ZO-1 and Occludin. Moreover, JWH-015 increase the expression of p-PKB and p-FoxO1 protein and decrease the expression of caveolin-1, which was inhibited by LY294002 or MPG. Taken together, all of these results suggested that JWH-015 could regulate the BSCB permeability and this effect could be related to $\mathrm{PI} 3 \mathrm{~K} / \mathrm{PKB} / \mathrm{FoxO} 1 /$ caveolin-1 signal pathway.

To study the role of $\mathrm{CB} 2$ receptors in $\mathrm{BSCB}$ function, we examine the effects and mechanism of CB2 agonist, JWH-015 on BSCB permeability in in vivo and in vitro models. We demonstrated that the permeability of BSCB was significantly decreased by JWH-015 at 1 day, 3 day and 7 day after SCII compared with the control group. And JWH-015 resulted in dose- and time-dependent augmentation of tightness of HBMECs. The results suggest a role for CB2 receptors in the protection of BSCB injury in SCII correspondence with other models of brain injury (Zarruk et al., 2012; Vendel et al., 2014). Together, these analyses provide compelling evidence that CB2 receptor agonist promotes and enhances BSCB integrity during ischemia-reperfusion. The protective mechanism of BSCB induced by JWH-015 should be further investigated.

Caveolin-1 is the principal marker of caveolae in endothelial cells. It plays an 
important role in physiological and pathological conditions of the BBB (Gu et al., 2012; Badaut et al., 2015) and is related to the diminished expression of tight junction-associated proteins and metabolic pinocytosis vesicles when the $\mathrm{BBB}$ is destroyed by outside invaders (Xie et al., 2014). Caveolin-1, which serves as a platform or medium for signal transduction, cooperates with several signal molecules by forming a complex. Silencing of caveolin-1 and disruption of caveolae can attenuate or remove pathological damage and even engender the opposite effects in the BBB (Gu et al., 2012). Our results showed that the protein expression levels of caveolin-1 in spinal cord tissue were significantly induced at day 1, day 3 and day 7 after reperfusion. Moreover, the pretreatment with JWH-015 greatly prevented the up-regulation of caveolin-1 induced by SCII (Fig. 6 A and C). This studies suggested that the role of caveolin-1 in the BSCB that may have profound implications for central nervous system ischemia disease. TJ are composed of complicated TJ proteins including transmembrane proteins (Occludin and Claudins), members of the peripheral membrane protein family (zonula occludens) and adhesion molecules, etc (Haseloff et al. 2014). Disfunctions of TJ, which represent BBB disruption, were most frequently seen in BBB during I/R (Rosenberg et al., 2012). Although various signaling mechanisms can influence BBB tightness, TJ proteins located between brain endothelial cells assure tightness of the BBB. Therefore, the amount of TJ proteins in HBMEC membrane fractions was evaluated after pretreatment with JWH-015. The results suggested that the protein expression levels of Occludin and ZO-1 in spinal cord tissue were significantly decreased at 1 day, 3 day and 7 day after SCII, which were significantly upregulated by JWH-015 pretreatment. Previous studies demonstrated caveolin-1 could negatively regulate tight junction-associated proteins (Gu et al., 2012). However, whether the change of TJ protein induced by JWH-015 is regulated by caveolin-1 is still unclear. All of these results suggested that JWH-015 might regulate the BSCB permeability and this effect could be related to paracellular and transcellular pathway.

FoxO1 is an important transcription factor involved in regulating the function of vascular endothelial cells. FoxO1 is normally located in the nucleus, where it can bind 
to promoter regions of target genes, like caveolin-1, and then regulate their expression (Ferdous et al., 2011). FoxO1 is an important effect factor of phosphoinositide-3 kinase/protein kinase $\mathrm{B}(\mathrm{PI} 3 \mathrm{~K} / \mathrm{PKB})$ signaling pathways and gene transcription regulation switch, the expression of target gene can be closed or started accompanied by FoxO1 phosphorylation and dephosphorylation (Herzog et al., 2008). And activation of Akt results in the inactivation of FoxOs which may mediate ischemic tolerance (Fukunaga et al., 2009). Activation of CB 2 receptor could stimulate the PI3K/PKB pathway ( $\mathrm{Li}$ et al., 2013). Based on above-mentioned results, we hypothesize that JWH-015 could decrease the permeability of BSCB through inhibiting the Akt/FoxO1/caveolin-1 signal pathway. Therefore, we examined the expression of p-FoxO1 and p-PKB protein at different time points after JWH-015 treatment. The results demonstrated that the phosphorylation level of FoxO1 and PKB began to increase at $12 \mathrm{~h}$ and reached a maximum at $24 \mathrm{~h}$. In addition, we demonstrated that the inhibitor of ROS or PI3K significantly attenuated the expression of p-FoxO1 and p-PKB and then increased the expression of caveolin-1 protein in HBMECs induced by JWH-015 treatment, suggesting that JWH-015 might regulate the expression of caveolin-1 protein via regulating ROS/PI3K/PKB/FoxO1 signal pathway.

In conclusion, the mechanism by which $\mathrm{CB} 2$ receptor agonist improves the BSCB dysfunction induced by SCII is complex. Our results provide a evidence that CB2 receptor agonist, JWH-015, improves the function of BSCB through paracellular and transcellular pathway. And pretreatment of pharmacological CB2 receptor ligands is a clinically feasible approach for protection against SCII in the future.

\section{Materials and methods}

\subsection{Materials}

DMEM and fetal bovine serum were purchased from Life technologies Corporation, USA. EBM-2 medium (\#190860, Lonza), Chemically Defined Lipid Concentrate

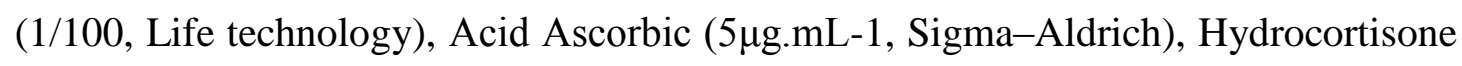
(1.4 $\mu \mathrm{M}$, Sigma-Aldrich), bFGF (1ng.mL-1, Sigma-Aldrich). A-gelatin, trypsin, l-glutamine, Horseradish peroxidase (HRP), N-2-mercaptopropionyl glycine (MPG), LY294002 and JWH-015 were obtained from Sigma-Aldrich Corporation, USA. 
Anti-ZO-1 and anti-Occludin antibodies were purchased from Life technology, USA. Anti-caveolin-1 antibody were purchased from BD Biotechnology. Anti-p-PKB and anti-p-FoxO1 antibody were purchased from Abcam Biotechnology.

\subsection{Experiments in vivo}

\subsubsection{Establishment of ischemia-reperfusion of spinal cord in rats}

Adult male Wistar rats (body weight 180-220g) were purchased from the Center for Experimental Animals of Shenyang Pharmaceutical University. All animal experiments were conducted in accordance with the National Institutes of Health Guide for the Care and Use of Laboratory Animals of the Chinese Academy of Sciences. All rats were anesthetized (10\% chloral hydrate, $3.5 \mathrm{ml} / \mathrm{kg}$, i.p.) and then were fixed on the experimental platform. The kidney, abdominal aorta and the bilateral renal artery were exposed. The abdominal aorta between the left and right renal artery was closed by noninvasive arterial clip for $30 \mathrm{~min}$, and then abdominal aortic pulsation disappearance was confirmed. Sham-operated group, sham surgery included general anesthesia, abdominal aorta and the bilateral renal artery were exposed but was not closed. Penicillin was injected intraperitoneally for connective 3 days.

\subsubsection{Treatment with JWH-015}

JWH-015 (1 mg/kg, i.p.), a CB2 receptor selective agonist, or vehicles (5\% DMSO) were injected in JWH-015 group and control group 20 minutes before ischemia, respectively. At day 1, day 3 and day 7 after reperfusion, rats were executed and spinal cord tissues of $\mathrm{L}_{2}-\mathrm{L}_{5}$ were extracted. And six rats from each group were used in the following experiments, respectively.

\subsubsection{Measurement of BSCB permeability by Evans blue leakage}

BSCB permeability was quantitatively evaluated by extravasation of Evans blue (EB) as a marker of albumin extravasation. Briefly, $2 \%$ EB in saline $(2 \mathrm{ml} / \mathrm{kg})$ was injected via tail vein in rats. Two hours after EB injection, rats in different group were deeply anesthetized with chloral hydrate and transcardially perfused until colorless perfusion fluid was obtained from the right atrium. Spinal cord tissue were weighed and 
immersed in formamide $(1 \mathrm{ml} / 100 \mathrm{mg})$ at $60{ }^{\circ} \mathrm{C}$ for $24 \mathrm{~h}$. The supernatant was obtained, and its optical density was determined with a spectrophotometer (at $620 \mathrm{~nm}$ ). The quantitative calculation of the dye content in the spinal cord tissue was based on the external standards dissolved in the same solvent (Fig.1).

\subsubsection{Fluorescence assays of Evans blue}

Rats for different time point groups were fixed by transaortic perfusion with saline, followed by perfusion with $4 \%$ paraformaldehyde. The spinal cord tissues in different group were cut in $10 \mu \mathrm{m}$ section at $-25^{\circ} \mathrm{C}$ using frozen-section machine. The images were analyzed using immunofluorescence microscopy (IX71, Olympus).

\subsubsection{Western blot analysis}

The effect of JWH-015 on Occludin and ZO-1 was analyzed via Western blots. Spinal cord tissue of $\mathrm{L}_{2}-\mathrm{L}_{5}$ from different time point groups were removed, respectively. Protein homogenates of spinal cord samples were prepared by rapid homogenization in lysis buffer. Samples were centrifuged at $17000 \mathrm{~g}$ for $20 \mathrm{~min}$ and Protein was quantified using the BCA protein assay kit (Bicin-choninic Acid, Kangwei Biotechnology, Inc) by Enzyme-labelled meter with $595 \mathrm{~nm}$. The Protein lysates (25 $\mu \mathrm{g}$ per lane for each sample) were fractioned on $10 \%$ NuPAGE Tris-Acetate gels. The Protein lysates (30 $\mu \mathrm{g}$ per lane for each sample) were fractioned and concentrated on 10\% NuPAGE Tris-Acetate gels, And then using iBlot Gel Transfer Device, we perform dry blotting of protein from mini gels with iBlot Gel Transfer Stacks for 7-9 min according to molecular weight of protein. The membranes were blocked in blocking buffer (5\% non-fat dairy milk dissolved in Tween-Tris-buffered saline, TTBS) for $2 \mathrm{hr}$ at room temperature. The blots were then incubated with rabbit polyclonal antibody anti-Occludin (Invitrogen, Life technology), anti-ZO-1 (Invitrogen, Life technology), anti-FoxO1 (1:800, thermo), anti-p-FoxO1(1:800, Abcam), anti-caveolin-1(1:1000, BD Biotechnology) anti- $\beta$-actin (dilution 1:20000; Proteintech, Inc.) overnight at $4^{\circ} \mathrm{C}$. The Occludin, ZO-1, p-FoxO1, FoxO1, caveolin-1 bands and $\beta$-actin on these immunoblots were visualized using enhanced chemiluminescence (ECL kit, Santa Cruz Biotechnology, Inc.). And $\beta$-actin represents a control procedure. The protein bands were scanned using Chemi Imager 
5500 V2.03 software, and IDVs were calculated by Fluor Chen 2.0 software and normalized with that of $\beta$-actin.

\subsection{Experiments in vitro}

\subsubsection{Establishment of BBB model in vitro}

Human brain microvascular endothelial cells (HBMECs) were cultured as described (Scott and Bicknell 1993). Cells were grown in an EBM-2 medium (Lonza) containing 10\% Fetal Bovine Serum (gibico, Life technology), Hydrocortisone

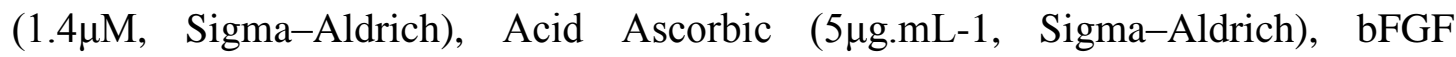
(1ng.mL-1, Sigma-Aldrich). HBMECs were cultured on the upper side of the transwell membrane. The cells were then allowed to achieve $80 \%$ confluency.

\subsubsection{OGD treatment}

To mimic spinal cord ischemia in vitro, OGD was performed. For OGD, medium was firstly removed from the cultures and then the cultures medium were rinsed twice with phosphate-buffered saline without $\mathrm{Ca}^{2+} / \mathrm{Mg}^{2+}$. Prior to OGD, cells were washed twice with PBS. The model group was exposed to sugar-free Earle's $(6.80 \mathrm{~g} / \mathrm{L} \mathrm{NaCl}$, $0.40 \mathrm{~g} / \mathrm{L} \mathrm{KCl}, 0.20 \mathrm{~g} / \mathrm{L} \mathrm{CaCl} 2,0.20 \mathrm{~g} / \mathrm{L} \mathrm{MgSO} 4 \cdot 7 \mathrm{H} 2 \mathrm{O}, 1.14 \mathrm{~g} / \mathrm{L} \mathrm{Na} 2 \mathrm{HPO} 4 \cdot 2 \mathrm{H} 2 \mathrm{O}$, and $2.20 \mathrm{~g} / \mathrm{L} \mathrm{NaHCO} 3$ ). JWH-015 was dissolved in dimethyl sulfoxide (DMSO) as vehicle and filtered with sugar-free Earle's and then divided into three groups $(1,10$, and $100 \mu \mathrm{M})$ according to the results of previous experiments. Afterward, cultures were placed into a three-gas $(\mathrm{O} 2, \mathrm{CO} 2$ and $\mathrm{N} 2)$ incubator at $37{ }^{\circ} \mathrm{C}$ with $1 \% \mathrm{O} 2$ for 24 h. The cell monolayers were split into the following groups: Normal group, OGD group, OGD+JWH-015 (1, 10, and $100 \mu \mathrm{M})$ groups, OGD+JWH-015 $(10 \mu \mathrm{M})+\mathrm{MPG}$ $(10 \mu \mathrm{M})$ group, OGD+JWH-015 $(10 \mu \mathrm{M})+\mathrm{LY} 294002(2 \mu \mathrm{M})$ group.

\subsubsection{Measurement of Transendothelial Electric Resistance}

Transendothelial electrical resistance (TEER) of HBMEC monolayers cultured on Transwell filters ((pore size, $0.4 \mu \mathrm{m})$ coated with $150 \mu \mathrm{g} / \mathrm{mL}$ of Cultrex Rat Collagen I.) was measured with a resistance meter (Millicell ERS-2, Millipore). The final TEER values were calculated as ohms per square centimeter $\left(\Omega \mathrm{cm}^{2}\right)$ by the surface area of the Transwell insert. 


\subsubsection{Horseradish Peroxidase Flux Measurement}

The HBMECs monolayers in Transwell inserts were cultured with OGD with or without JWH-015 pretreatment and then transferred to a new 24-well plate. Horseradish peroxidase (HRP, $0.5 \mu \mathrm{M}$, Sigma-Aldrich) in serum-free DMEM was added into the upper compartment of the Transwell system. And then the media from the lower chamber was collected and the content of HRP in the samples was assayed colorimetrically. The HRP flux was expressed as picomoles passed per square centimeter surface area (Xie et al. 2011).

\subsubsection{Western blot analysis}

The effect of JWH-015 on ZO-1, Occludin, caveolin-1, p-FoxO1 and p-PKB protein in HBMECs after OGD with or without JWH-015 pretreatment was analyzed via Western blotting. And The detailed process has been described in 4.2.5 .

\subsubsection{Immunofluorescence Assays}

The HBMEC monolayers grown on 24-well plate were permeabilized with $0.2 \%$ Triton X-100 in PBS on ice for two minutes. And fixed with 3\% paraformaldehyde for 30 minutes and permeabilized with $0.05 \%$ Triton X-100 for five minutes. After blocking with $5 \%$ bovine serum albumin in PBS, cells were incubated with primary antibodies against Occludin (1:25) and ZO-1 (1:30) to assess the expression of Occludin and ZO-1. The cells were incubated with rhodamine-labeled /FITC-goat anti-rabbit (diluted 1:1000, Life technology) for $1 \mathrm{hr}$ at room temperature. The images were analyzed using immunofluorescence microscopy .

\subsection{Statistical analysis}

All values are expressed as the mean \pm SD. One-way analysis of variance (ANOVA) was used to compare the difference among groups in the measurements of TJ protein, caveolin-1, p-PKB, FoxO1, p-FoxO1 expression levels. Dunnett's post hoc tests were applied to compare specific difference between groups if there was a great difference showed by ANOVA. For other measurements, the data were assessed using paired Student's t test. $P<0.05$ was considered significant difference.

\section{Acknowledgments}

This work was supported by National Science Foundation for China, No.81100924; 
National Science Foundation of Liaoning Province for excellent talents, No.2014021016.

\section{References}

Arevalo-Martin A, Garcia-Ovejero D, Sierra-Palomares Y, Paniagua-Torija B, Gonzalez-Gil I, Ortega-Gutierrez S, Molina-Holgado E. 2012. Early endogenous activation of CB1 and CB2 receptors after spinal cord injury is a protective response involved in spontaneous recovery. PLoS One. 7(11):e49057.

Badaut J, Ajao DO, Sorensen DW, Fukuda AM, Pellerin L. 2015. Caveolin expression changes in the neurovascular unit after juvenile traumatic brain injury: signs of blood-brain barrier healing? Neuroscience. 285:215-26.

Blixt J, Svensson M, Gunnarson E, Wanecek M. 2015. Aquaporins and blood-brain barrier permeability in early edema development after traumatic brain injury. Brain Res. Mar 11. pii: S0006-8993(15)00185-7.

Choi IY, Ju C, Anthony Jalin AM, Lee da I, Prather PL, Kim WK. 2013. Activation of cannabinoid CB2 receptor-mediated AMPK/CREB pathway reduces cerebral ischemic injury. Am J Pathol. 182(3):928-39.

England TJ, Hind WH, Rasid NA, O'Sullivan SE. Cannabinoids in experimental stroke: a systematic review and meta-analysis. J Cereb Blood Flow Metab. 2014 Dec 10. doi: 10.1038/jcbfm.2014.218.

Ferdous A., Morris J., Abedin M.J., Collins S., Richardson J.A., Hill J.A.. 2011. Forkhead factor FoxO1 is essential for placental morphogenesis in the developing embryo. Proc Natl Acad Sci U S A. $108(39): 16307-12$

Fukunaga K, Shioda N. 2009. Pathophysiological relevance of forkhead transcription factors in brain ischemia. Adv Exp Med Biol.665:130-42.

Gu Y, Zheng G, Xu M, Li Y, Chen X, Zhu W, Tong Y, Chung SK, Liu KJ, Shen J. 2012. Caveolin-1 regulates nitric oxide-mediated matrix metalloproteinases activity and blood-brain barrier permeability in focal cerebral ischemia and reperfusion injury. $\mathrm{J}$ Neurochem. 
120(1):147-56.

Haseloff RF, Dithmer S, Winkler L, Wolburg H, Blasig IE. 2014. Transmembrane proteins of the tight junctions at the blood-brain barrier: Structural and functional aspects. Semin Cell Dev Biol. Nov 26. pii: S1084-9521(14)00314-0.

Herzog S, Hug E, Meixlsperger S, Paik JH, DePinho RA, Reth M, Jumaa H. 2008. SLP-65 regulates immunoglobulin light chain gene recombination through the PI(3)K-PKB-Foxo pathway. Nat Immunol. 9(6):623-631.

Hind WH, Tufarelli C, Neophytou M, Anderson SI, England TJ, O'Sullivan SE. 2015. Endocannabinoids modulate human blood-brain barrier permeability in vitro. Br J Pharmacol. Feb 5. doi: 10.1111/bph.13106.

Huang P, Zhou CM, Qin-Hu, Liu YY, Hu BH, Chang X, Zhao XR, Xu XS, Li Q, Wei XH, Mao XW, Wang CS, Fan JY, Han JY. 2012. Cerebralcare Granule attenuates blood-brain barrier disruption after middle cerebral artery occlusion in rats. Exp Neurol. 237(2):453-63.

Li Q, Wang F, Zhang YM, Zhou JJ, Zhang Y. 2013. Activation of cannabinoid type 2 receptor by JWH133 protects heart against ischemia/reperfusion-induced apoptosis. Cell Physiol Biochem. 31(4-5):693-702.

Li XQ, Lv HW, Wang ZL, Tan WF, Fang B, Ma H. 2015. MiR-27a ameliorates inflammatory damage to the blood-spinal cord barrier after spinal cord ischemia: reperfusion injury in rats by downregulating TICAM-2 of the TLR4 signaling pathway. J Neuroinflammation. 12(1):246.

Moretti R, Pansiot J, Bettati D, Strazielle N, Ghersi-Egea JF, Damante G, Fleiss B, Titomanlio L, Gressens P. 2015. Blood-brain barrier dysfunction in disorders of the developing brain. Front Neurosci. 9:40

Mougeolle A, Poussard S, Decossas M, Lamaze C, Lambert O, Dargelos E. 2015. Oxidative stress induces caveolin 1 degradation and impairs caveolae functions in skeletal muscle cells. PLoS One. 10(3):e0122654.

Nag S, Venugopalan R, Stewart DJ. 2007. Increased caveolin-1 expression precedes decreased expression of occludin and claudin-5 during blood-brain barrier breakdown. Acta Neuropathol. 114(5):459-469.

Ramirez SH, Haskó J, Skuba A, Fan S, Dykstra H, McCormick R, Reichenbach N, Krizbai I, Mahadevan A, Zhang M, Tuma R, Son YJ, Persidsky Y. 2012. Activation of cannabinoid receptor 
2 attenuates leukocyte-endothelial cell interactions and blood-brain barrier dysfunction under inflammatory conditions.J Neurosci. 32(12):4004-16.

Rosenberg GA. 2012. Neurological diseases in relation to the blood-brain barrier. J Cereb Blood Flow Metab. 32(7):1139-51.

Shin T. 2007. Increases in the phosphorylated form of caveolin-1 in the spinal cord of rats with clip compression injury. Brain Res. 1141:228-34.

Vendel E, de Lange EC. 2014. Functions of the CB1 and CB 2 receptors in neuroprotection at the level of the blood-brain barrier. Neuromolecular Med. 16(3):620-42.

Xie L, Xue X, Taylor M, Ramakrishnan SK, Nagaoka K, Hao C, Gonzalez FJ, Shah YM. 2014. Hypoxia-inducible factor/MAZ-dependent induction of caveolin-1 regulates colon permeability through suppression of occludin, leading to hypoxia-induced inflammation. Mol Cell Biol. 34(16):3013-23.

Yu SJ, Reiner D, Shen H, Wu KJ, Liu QR, Wang Y. 2015. Time-Dependent Protection of CB2 Receptor Agonist in Stroke. PLoS One. 10(7):e0132487.

Zarruk JG, Fernández-López D, García-Yébenes I, García-Gutiérrez MS, Vivancos J, Nombela F, Torres M, Burguete MC, Manzanares J. 2012. Cannabinoid type 2 receptor activation downregulates stroke-induced classic and alternative brain macrophage/microglial activation concomitant to neuroprotection. Stroke. 43(1):211-9.

Zhao YL, Song JN, Zhang M. 2014. Role of caveolin-1 in the biology of the blood-brain barrier. Rev Neurosci. 25(2):247-54.

Zhong Y, Smart EJ, Weksler B, Couraud PO, Hennig B, Toborek M. 2008. Caveolin-1 regulates human immunodeficiency virus-1 Tat-induced alterations of tight junction protein expression via modulation of the Ras signaling.J Neurosci. 28(31):7788-96.

Fig. 1. The permeability of blood-spinal cord barrier (BSCB) was assessed by Evans blue (EB) extravasation ( $\mu \mathrm{g} / \mathrm{g}$ spinal cord tissue) in different time point groups (A). Data are presented as mean \pm S.D. ( $n=6$, each). ${ }^{*} P<0.05,{ }^{* *} P<0.01$ vs. SCII group. Immunofluoresence localization of EB in the vascular spinal cord in rats. B: SCII 1 day group; C: SCII 3 day group; D: SCII 7 day group; E: JWH-015+SCII 1 day group; F: JWH-015+SCII 3 day group; G: JWH-015+SCII 7 day group.

Fig. 2. Effects of $\mathrm{JWH}-015$ on $\mathrm{ZO}-1$ and Occludin protein in the $\mathrm{L}_{2}-\mathrm{L}_{5}$ site of spinal cord 
at day 1, day 3 and day 7 after SCII. (A) Lane 1: SCll group for 1 day; lane 2: JWH-015+SCII group for 1 day; lane 3: SCII group for 3 day; lane 4: JWH-015+SCII group for 3 day; lane 5: SCll group for 7 day; lane 6: JWH-015+SCII group for 7 day; Relative integrated density value (IDV) analysis of ZO-1 (B) and Occludin protein (C) (n=6, each). ${ }^{* *} P<0.01$ vs. SCll group. (D) Lane 1: Sham-operated group for 1 day; lane 2: JWH-015+Sham-operated group for 1 day; lane 3: Sham-operated group for 3 day; lane 4: JWH-015+Sham-operated group for 3 day; lane 5: Sham-operated group for 7 day; lane 6: JWH-015+Sham-operated group for 7 day. Relative integrated density value (IDV) analysis of ZO-1 (E) and Occludin protein (F) (n=6, each). ${ }^{* *} P<0.01$ vs. SCII group.

Fig. 3. Effects of JWH-015 on ZO-1 protein in the gray matter of spinal cord at day $1(A, D)$, day $3(B, E)$ and day $7(C, F)$ after SCII by immunohistochemical method. A,B,C: SCII group; D,E,F: JWH-015+ SCII group; Mean Optical density value analysis of ZO-1 protein (G) ( $n=6$, each). ${ }^{*} P<0.05$ vs.SCII group. Scale bar: $20 \mu \mathrm{m}$.

Fig. 4. Effects of JWH-015 on Occludin protein in the gray matter of spinal cord at day $1(A, D)$, day $3(B, E)$ and day $7(C, F)$ after SCIl by immunohistochemical method. A,B,C: SCII group; D,E,F: JWH-015+ SCII group; Mean Optical density value analysis of Occludin (G) (n=6, each). ${ }^{*} P<0.05,{ }^{* *} P<0.01$ vs. SCIl group. Scale bar: $20 \mu \mathrm{m}$.

Fig. 5. Effects of JWH-015 on Caveolin-1 protein in the $L_{2}-L_{5}$ spinal cord site at day 1 , day 3 and day 7 after SCII. Lane 1: Sham-operated group for 1 day; lane 2: SCII group for 1 day; lane 3: JWH-015+SCII group for 1 day; lane 4: Sham-operated group for 3 day; lane 5: SCII group for 3 day; lane 6: JWH-015+SCII group for 3 day; lane 7: sham-operated group for 7 day; lane 8: SCII group for 7 day; lane 9: JWH-015+SCII group for 7 day; (A) Representative Western blots illustrating differences in Caveolin-1. Relative integrated density value (IDV) analysis of Caveolin-1 (B) (n=6, each). ${ }^{* *} P<0.01$ vs. SCII group.

Fig.6.The permeability of blood-brain barrier (BBB) in vitro was assessed by transendothelial electric resistance (TEER) and horseradish peroxidase (HRP) flux in different groups. The effects of JWH-015 (10, 20, $100 \mu \mathrm{M})$ on TEER (A) and HRP flux (B) in HBMECs. Data are presented as mean \pm S.D. ( $n=6$, each). ${ }^{\star \star} P<0.01$ vs. SCIl group.

Fig. 7 Effects of JWH-015 on ZO-1 and Occludin protein in human brain microvascular 
endothelial cells after OGD. Lane 1:Normal group; Lane 2: OGD group; lane 3: JWH-015+OGD group. (A) Representative Western blots illustrating differences in ZO-1 and Occludin. Relative integrated density value (IDV) analysis of ZO-1 (B) and Occludin protein (C) ( $n=6$, each). ${ }^{* *} P<0.01$ vs. Normal group. ${ }^{\#} P<0.05$ vs. SCII group.

Fig. 8. Immunofluoresence localization of ZO-1 (A and B) and Occludin (C and D) protein in human brain microvascular endothelial cells (HBMECs) in OGD group and JWH-015+OGD group. A and C: OGD group; B and D: JWH-015+OGD group. And the reduction of ZO-1 Occludin protein induced by SCII was greatly inhibited by JWH-015 pretreatment. Scale bar: $20 \mu \mathrm{m}$.

Fig. 9. Effects of JWH-015 on Caveolin-1 protein in human brain microvascular endothelial cells after OGD. Lane 1:Normal group; Lane 2: OGD group; lane 3: JWH-015+OGD group. (A) Representative Western blots illustrating differences in Caveolin-1. Relative integrated density value (IDV) analysis of Caveolin-1 protein (B) $(n=6$, each). ${ }^{* *} P<0.01$ vs. Normal group. ${ }^{\# \#} P<0.05$ vs. SCII group.

Fig. 10. Effects of JWH015 with or without ROS inhibitor or PI3K inhibitor on p-FoxO1 and p-PKB protein expression in human brain microvascular endothelial cells (HBMECs) after OGD for $24 \mathrm{hr}$. Lane 1: OGD group; lane 2: JWH-015+OGD group; Lane 3: MPG+JWH-015+OGD group; Lane 4: LY294002+JWH-015+OGD group; Representative Western blots illustrating differences in the bands of p-FoxO1 (A) and p-PKB protein (B). Changes of relative integrated density value (IDV) of p-FoxO1 (C) and p-PKB protein (D) ( $n=6$, each). ${ }^{* *} P<0.01$ vs. OGD group, ${ }^{\# \#} P<0.01$ vs. JWH-015+OGD group. 
Figure 1
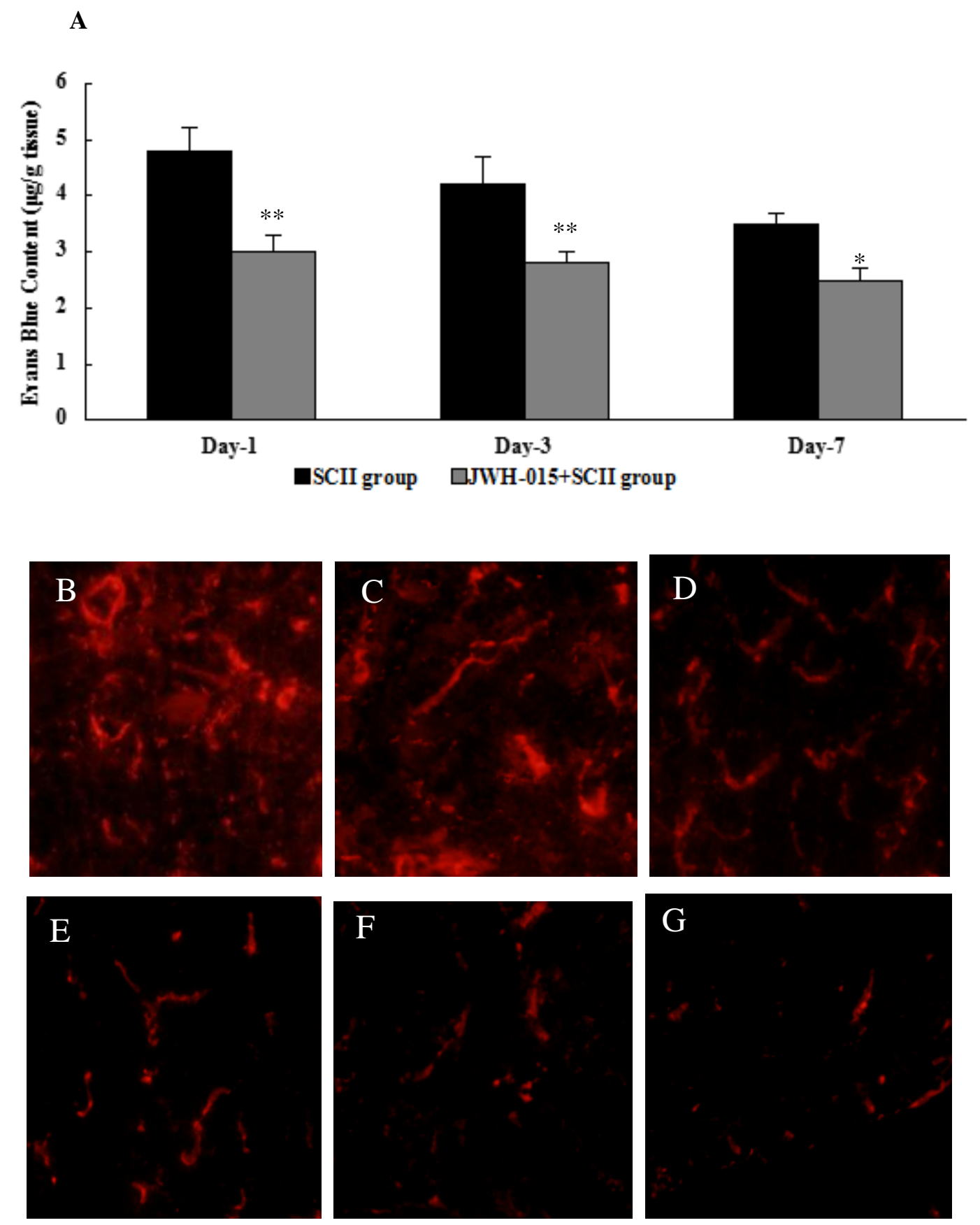

G

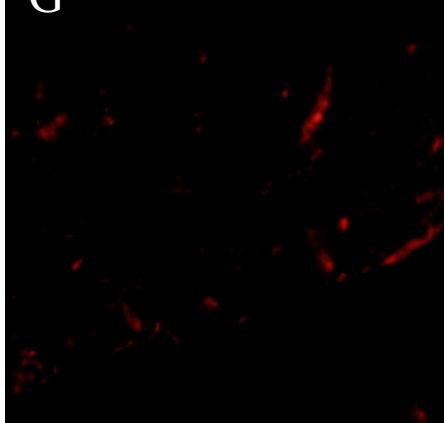




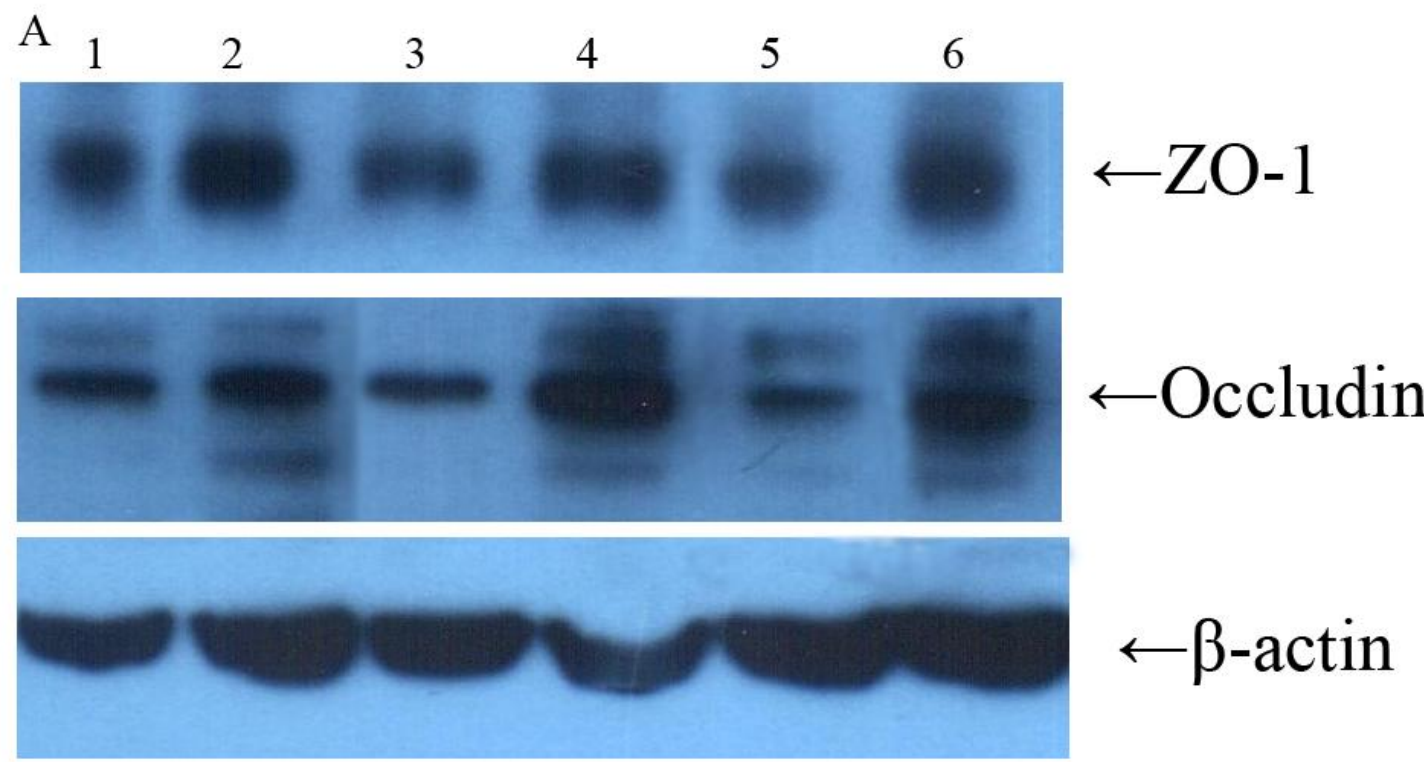

B

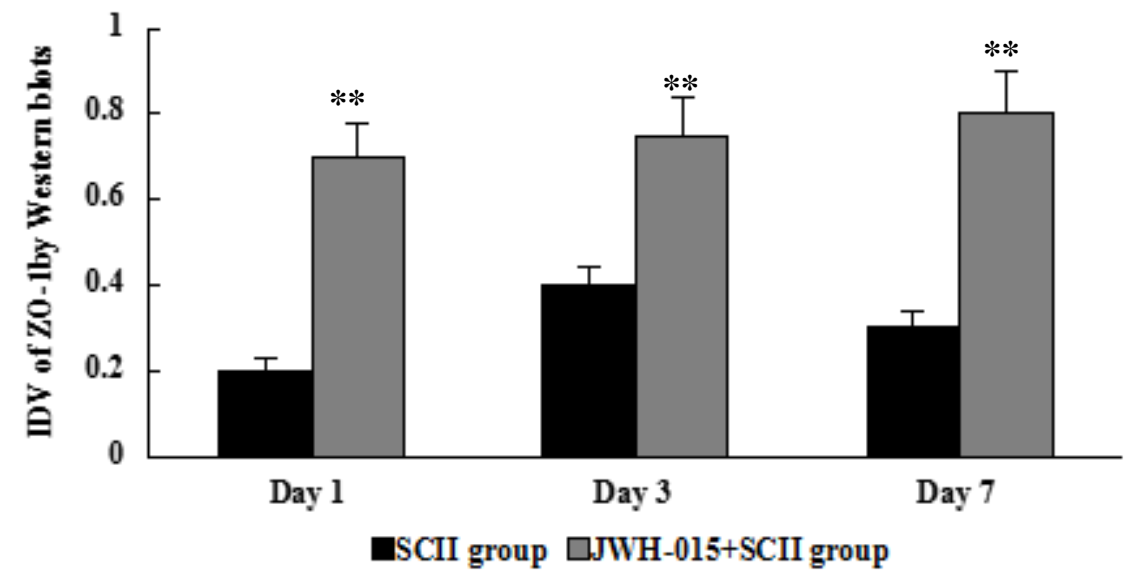

C

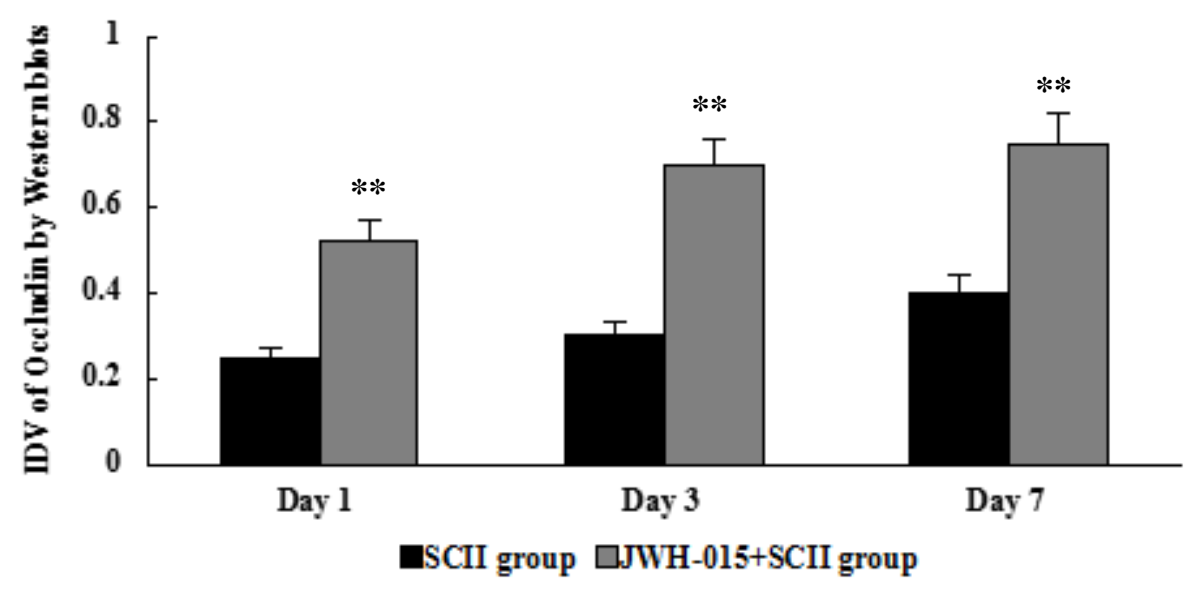


D

$\begin{array}{llllll}1 & 2 & 3 & 4 & 5 & 6\end{array}$
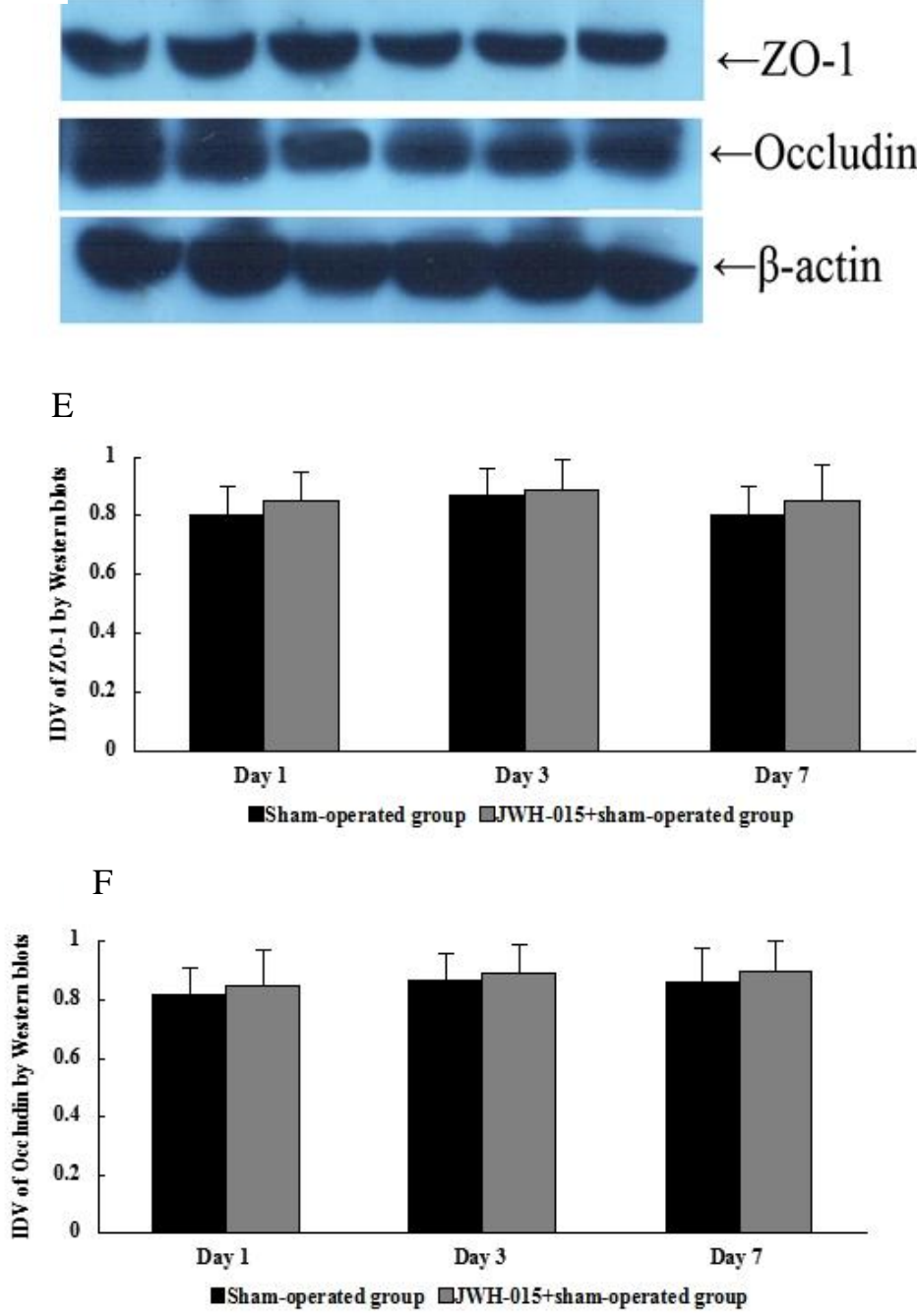
Figure 3
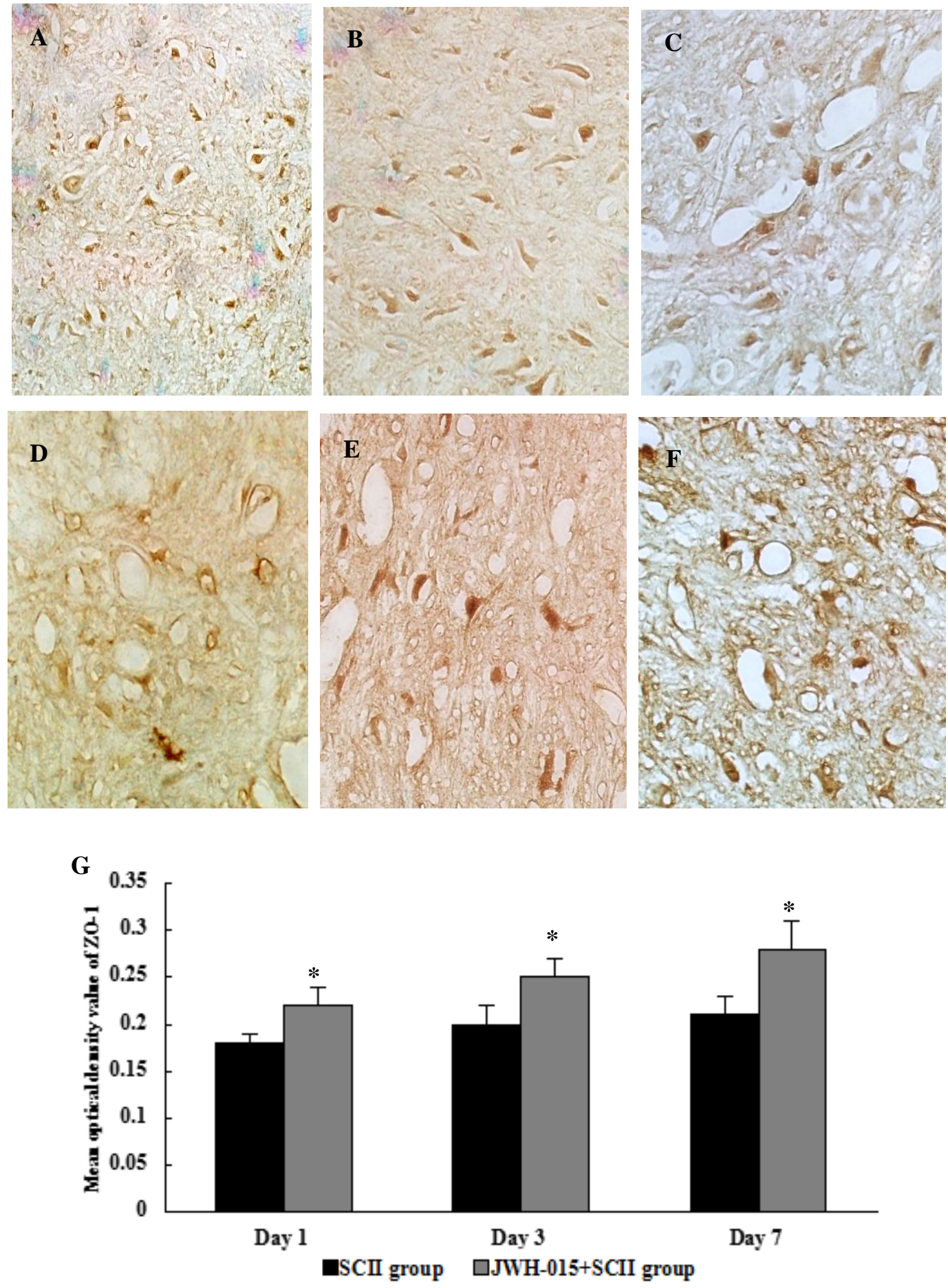
Figure 4
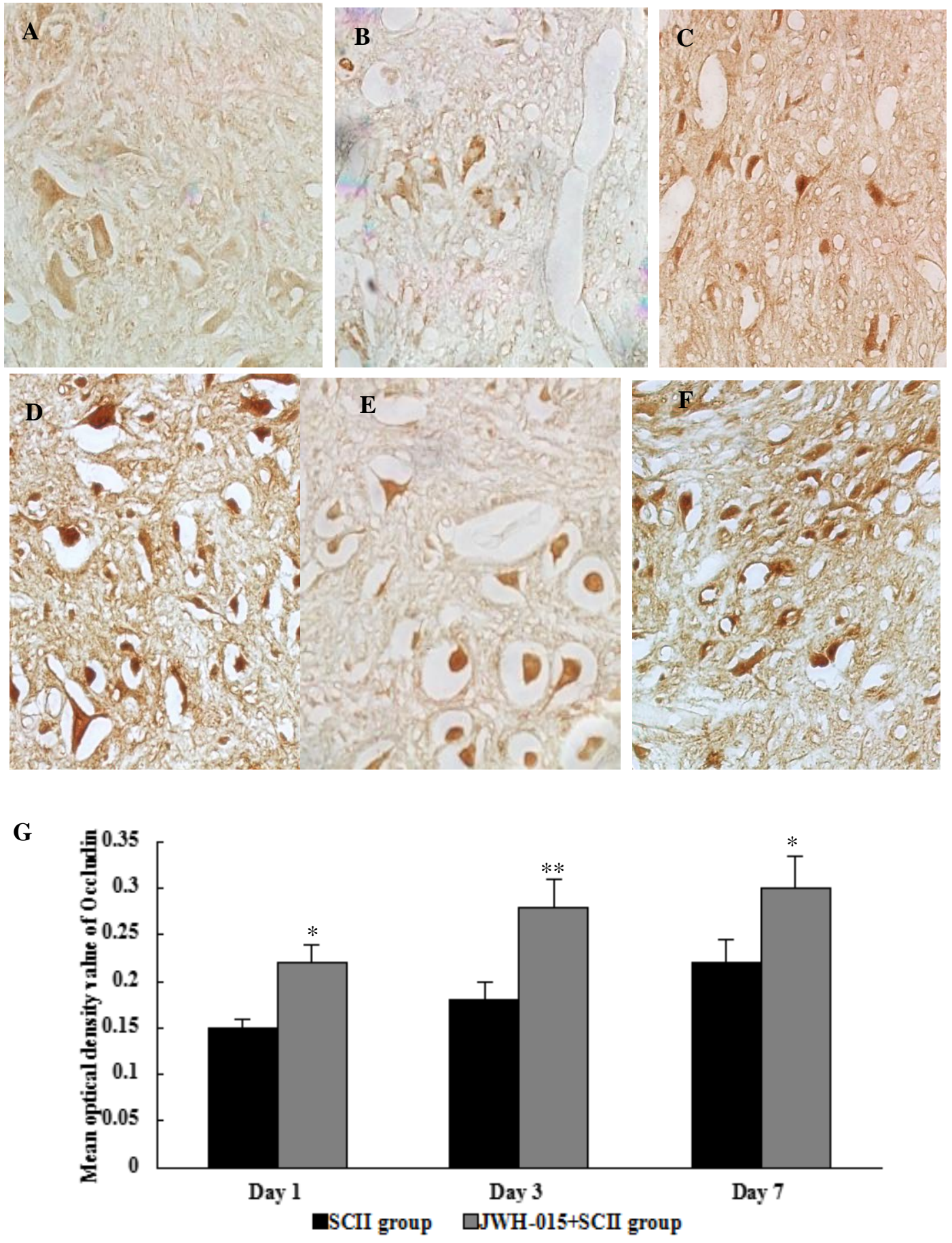
Figure 5

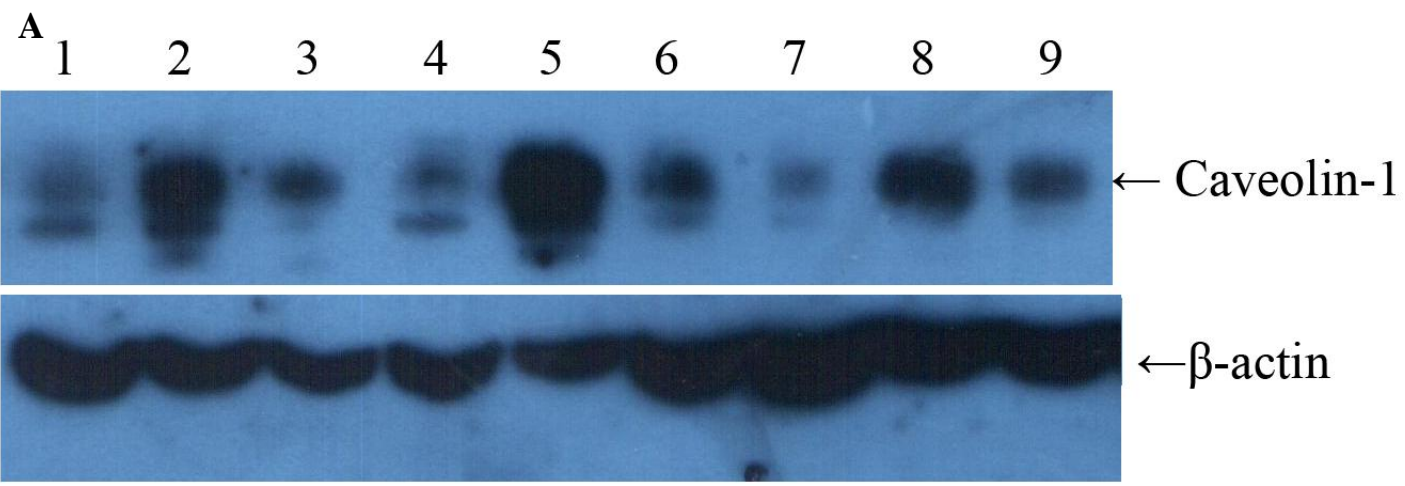

B

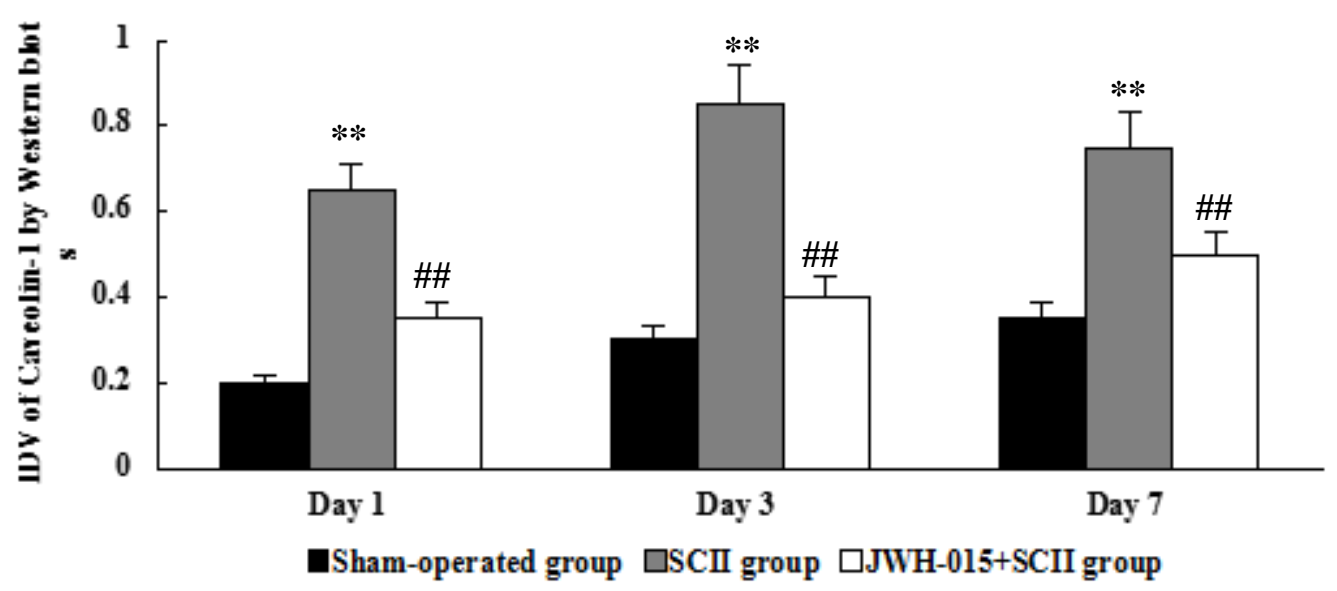


Figure 6
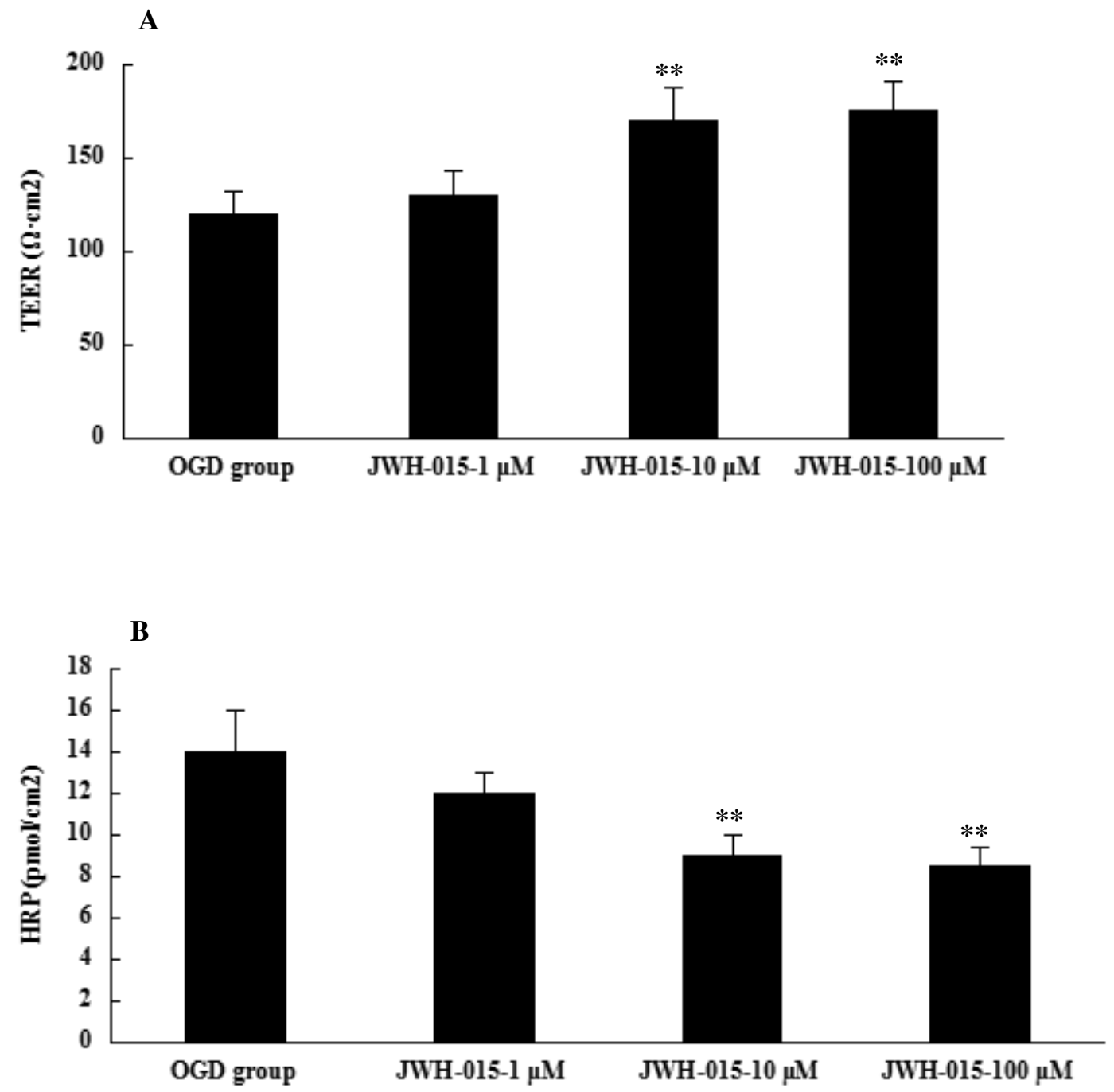
Figure 7

$\begin{array}{lll} & 1 & 2\end{array}$

$\leftarrow$ Occludin

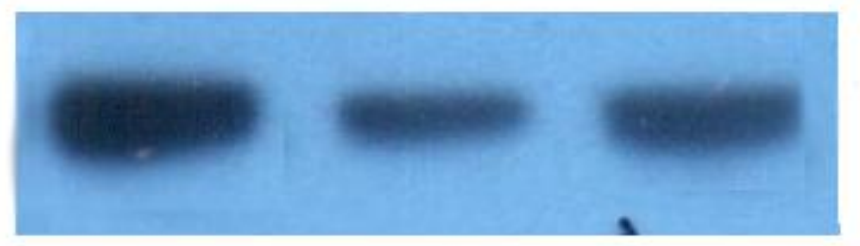

$\leftarrow \mathrm{ZO}-1$

$\leftarrow \beta$-actin

B
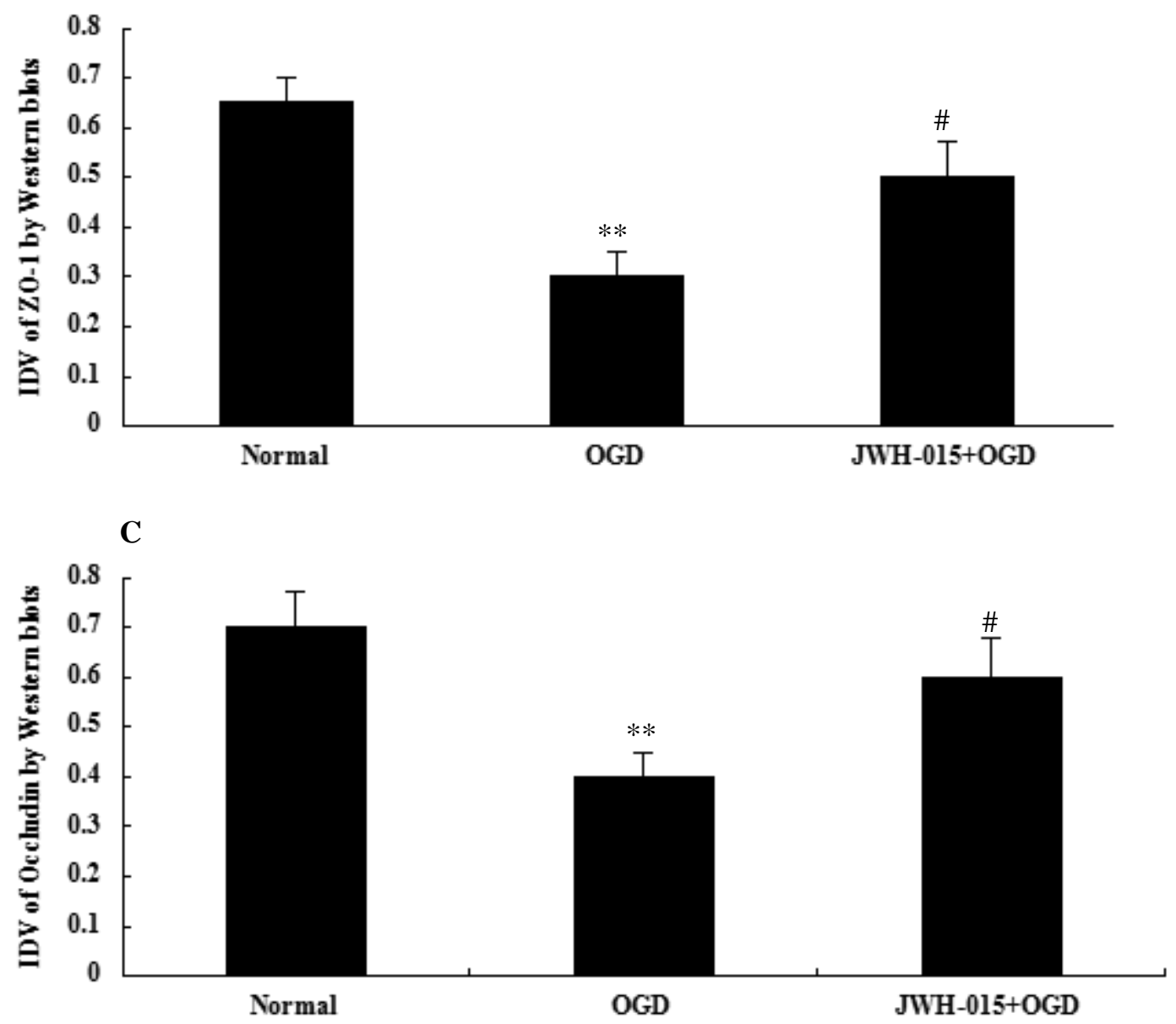

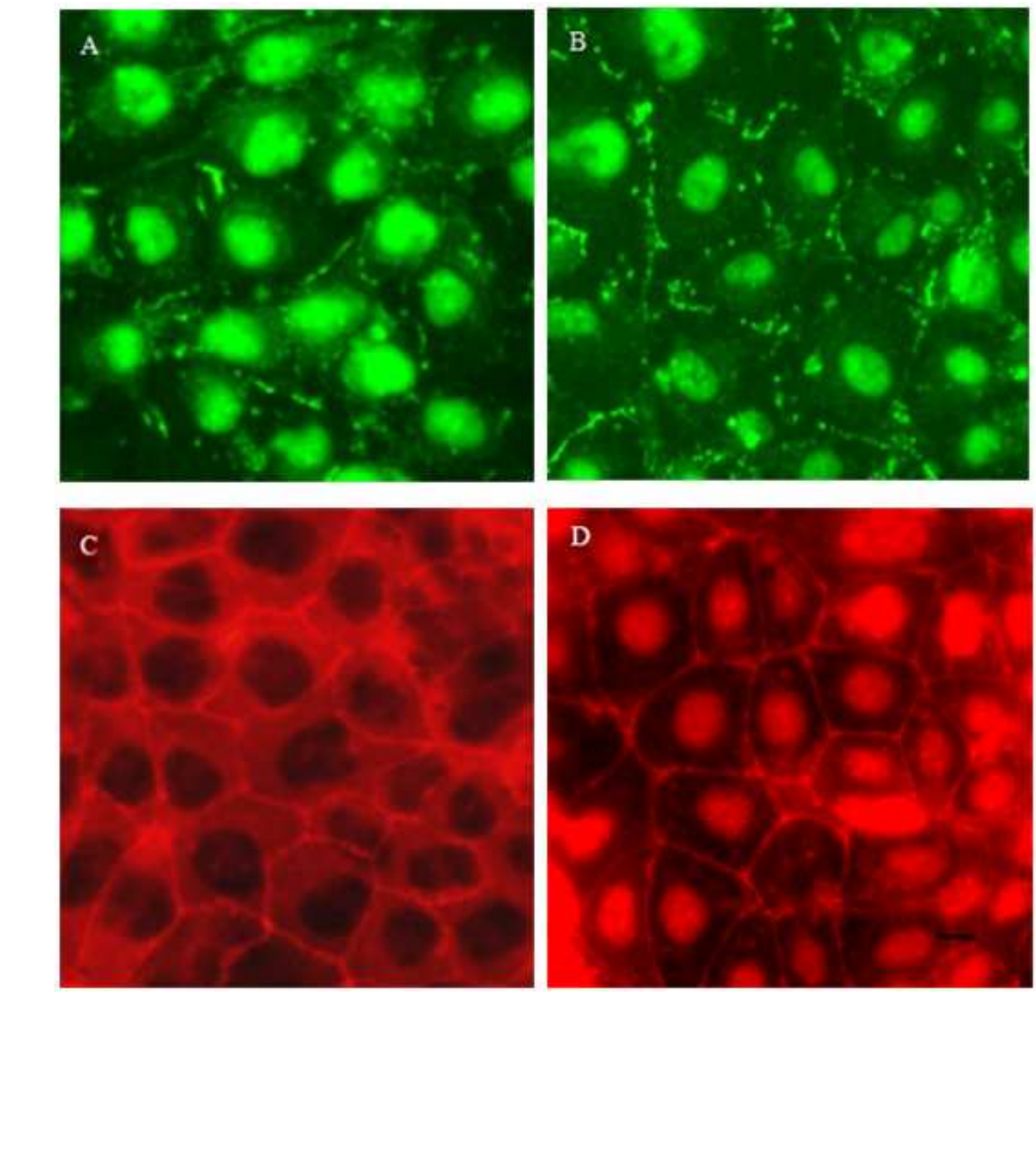

D

Figure 8

\section{D}

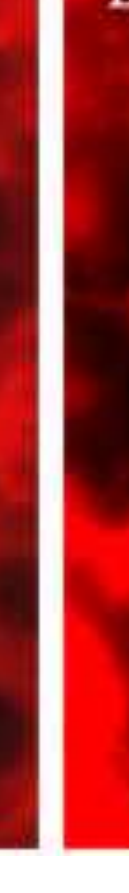
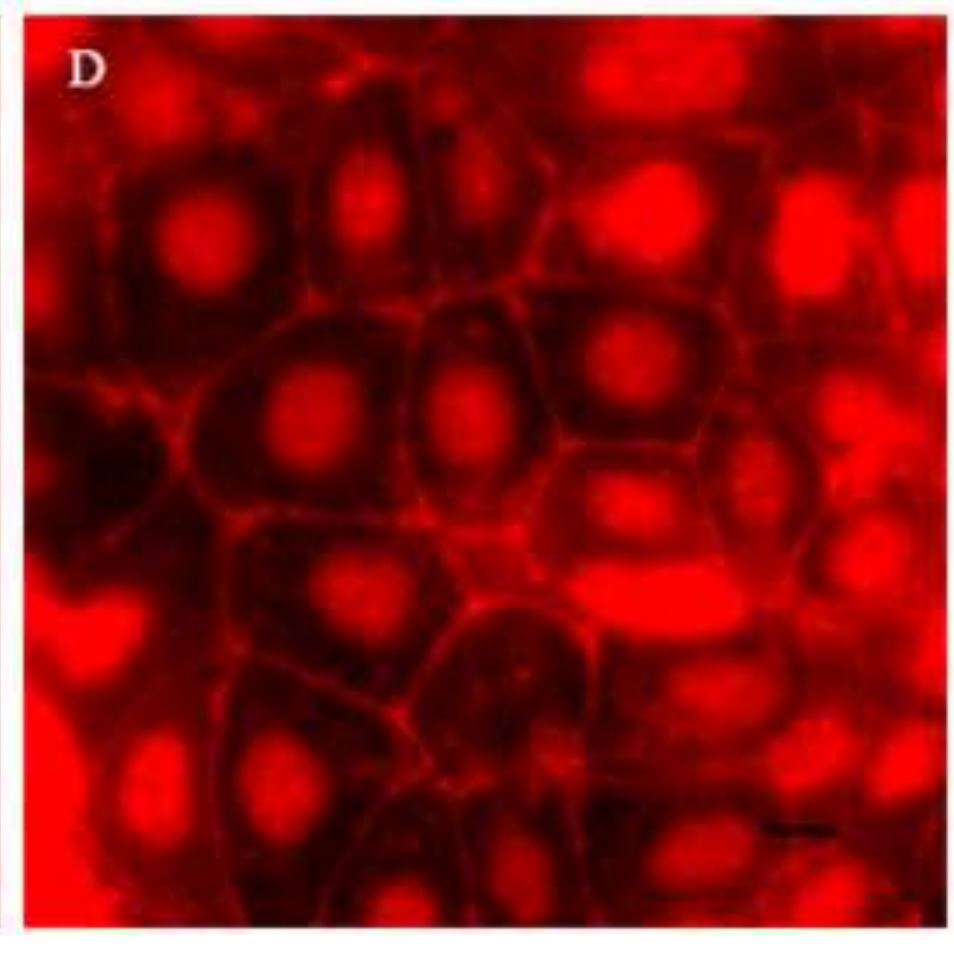
Figure 9

$\begin{array}{lll}\text { A } & 2 & 3\end{array}$

$\leftarrow$ Caveolin-1

$\leftarrow \beta$-actin

B
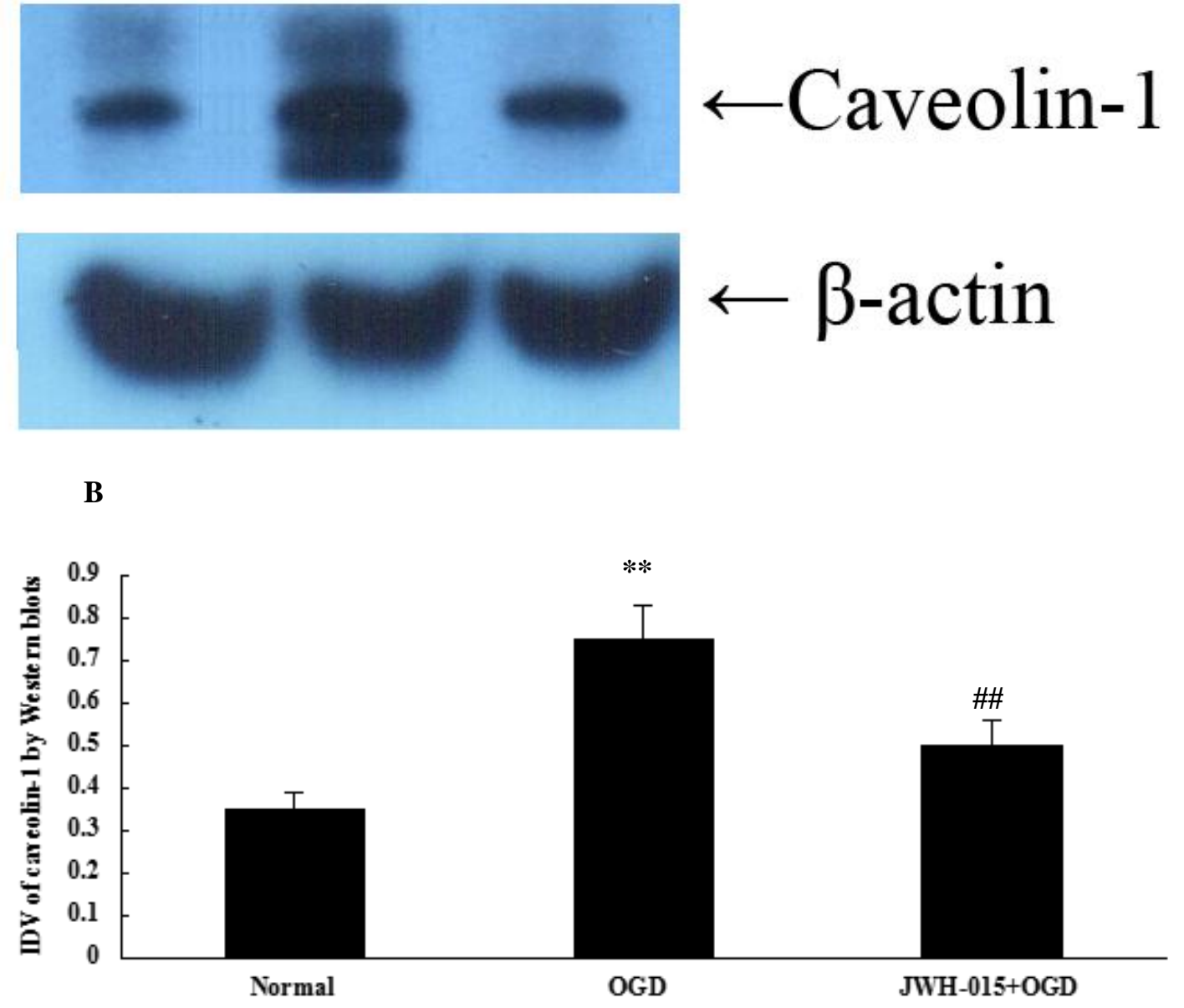
Figure 10
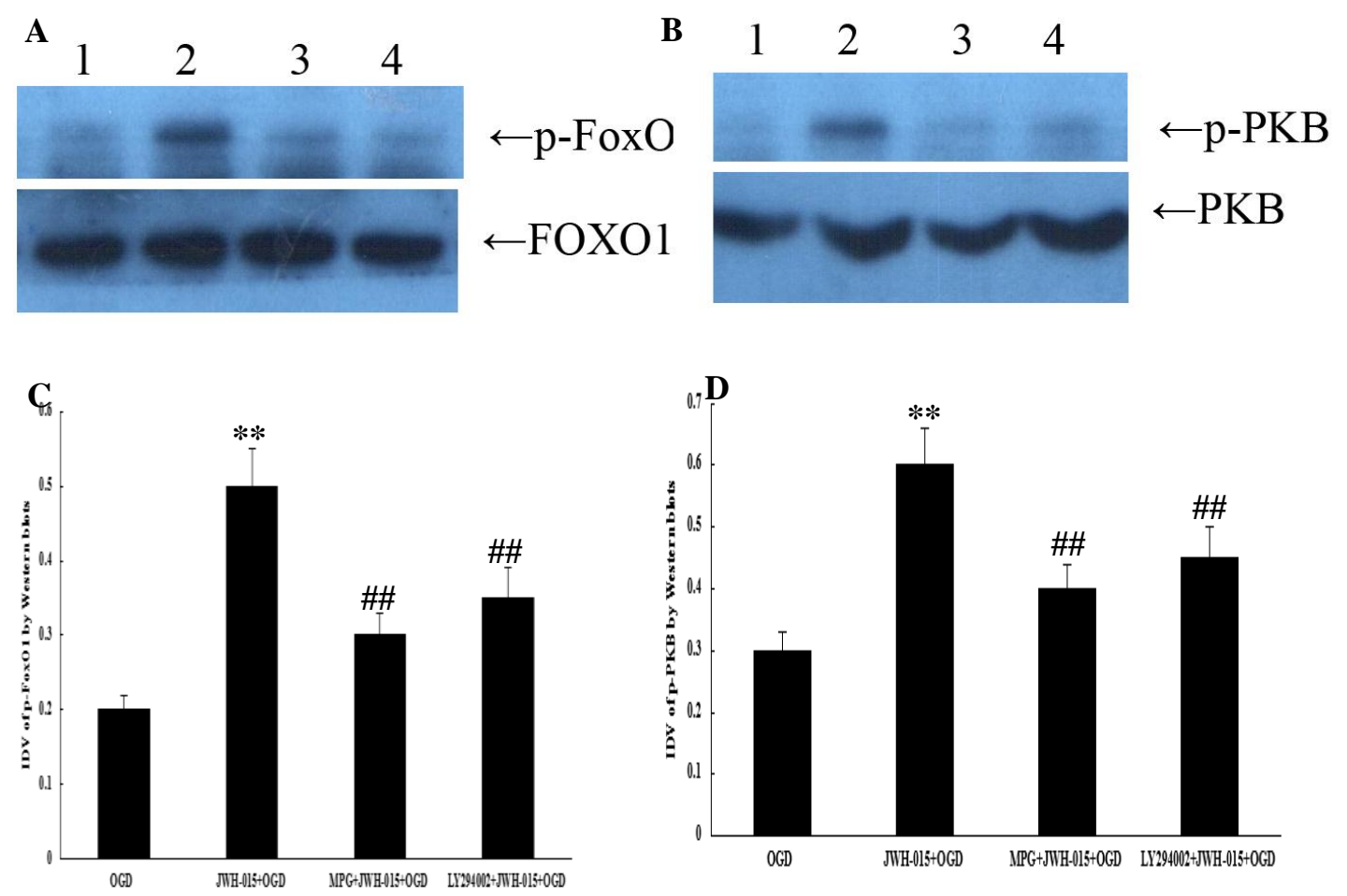